\title{
Resilience Assessment for HDG under Extreme Events
}

\author{
Saiyi Wang ${ }^{1}$, Weiguo $\mathrm{He}^{1}$, Haodong Shen ${ }^{2}$, Tangyun $\mathrm{Xu}^{2}$, Shichao Zhou ${ }^{3}$, Jie $\mathrm{Chen}^{2}$ and Yingying Zhao ${ }^{2}$ \\ ${ }^{1}$ State Grid Shanghai Pudong Electric Power Company, Shanghai, 200120, China \\ ${ }^{2}$ State Grid Shanghai Energy Internet Research Institute Co. Ltd, Shanghai, 200120, China \\ ${ }^{3}$ Department of Electrical Engineering, Shanghai Jiaotong University, Shanghai, 201100, China
}

\begin{abstract}
The resilience assessment method of hybrid AC-DC distribution grid is the key and basis of measuring the tolerance of the distribution network in face of extreme events and testing the effect of the resilience improvement strategy of the distribution network. This paper fully considers the characteristics of different extreme natural disasters and proposes a targeted resilience assessment method. For natural disasters, the fault rate model of the line under the influence of the disaster is constructed, and the fault set is generated based on Monte Carlo sampling. According to the operating characteristics, a set of evaluation indicators focusing on the core characteristics of resilience, including capability of strain, defense and recoverability, is constructed. The objective weight of each indicator and the comprehensive assessment results are obtained based relatively on the entropy weight method and the approximate ideal solution sorting method.
\end{abstract}

\section{Introduction}

Hybrid AC/DC distribution grid (HDG) combines the characteristics and advantages of $\mathrm{AC}$ and DC distribution grid, which can realize the output scheduling of distributed power within the range of wide-area power flow, and is closer to the power supply demand of load ${ }^{[1]}$. Not only the advantages of traditional AC distribution network are retained, but also the development bottlenecks of AC distribution network are effectively removed. HDG will surely become one of the main structural forms of the smart distribution network in the future. There are still key technical issues that need to be resolved before widely construction and application of HDG, one of which is the resilience improvement strategy in the face of extremely events with small probability and high risk.

China is located at the junction of the three major plates, with a continental coastline of 18,000 kilometers. The special geographical location and climatic environment have caused frequent and severe disturbances of natural disasters in most parts of China, such as extreme typhoons, heavy rains, and lightning strikes. The extreme natural disasters have caused tremendous impact and destruction on the transmission and distribution equipment in the landing area. Resilience was first proposed and introduced into the field of ecosystem research by Canadian ecologist C. S. Holling. The concept was then gradually extended to aspect of power systems and distribution networks as an evaluation index of the performance of the distributed networks before and after the occurrence of small-probability highrisk extreme events ${ }^{[2]}$. The core characteristics of resilience contain capability of strain, defense recoverability. Capability of strain refers to the ability of predicting the evolution and impacts and making corresponding preparatory and preventive measures before a large-scale disturbance event. Capability of defense is that the distribution network has the ability of making emergency response to incidents by adjusting flexible resources, thus it can reduce the impact and interference from incidents and maintain a high operation level as much as possible. Recoverability represents the ability of quickly starting the repair mechanism to return to normal operating state after disturbances, therefore minimizing the duration of the impact on the failure.

The concept of "generalized resilience" was proposed based on the actual demand and operating experience of the Chinese power grid [3], adding perception, learning ability and coordination to the original three core features. Many researches have carried out quantitative assessments of the resilience around the core characteristics and put forward a series of practical evaluation indicators [4]. Taking into account the uncertainty of the evolution of the temporal and spatial characteristics of the extreme events, some researchers analyzed the impact of extreme events on the distribution network lines, and built a multi-stage uncertain fault set for distribution network lines. Literature [5] comprehensively analyzed the characteristics of typical natural disasters and their impact on distribution network line faults. Literature [6] constructed the mapping between typhoon wind speed and line failure rate. All these researches have provided a solid foundation for the research on the resilience improvement strategy of the distribution network.

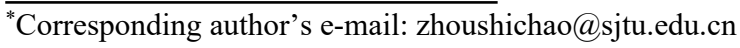


However, current assessment methods of distribution network resilience, such as simulation, analytical and statistical methods, are mostly post-analysis. It means that the economic losses and social adverse effects are only evaluated after extreme events happened. This paper focuses on extreme event such as natural disasters, among which two typical scenarios, including extreme ice and snow weather and typhoon are considered. A pre-resilient assessment method of HDG based on the characteristics of extreme events is proposed to provide assistance for operators in measuring the resilience performance of distribution networks in terms of capability of strain, defense, and recoverability before extreme events.

\section{Fault analysis of HDG considering the characteristics of extreme events}

Overhead lines are composed of wires, towers, insulators and other supporting equipment. Considering that the line faults are mainly caused by the destruction of wires and towers, and assuming that the failures of wires and towers are independent [7], the line failure rate expression can be obtained.

$$
p_{i j}^{f a u l t}=1-\prod_{k=1}^{m}\left(1-p_{i j, p, k}^{f a u l t}\right) \prod_{k=1}^{n}\left(1-p_{i j, c, k}^{f a u l t}\right)
$$

Where $p_{i j}^{\text {fault }}$ represents the failure rate of the line $i j$, $m$ represents the number of supporting towers of the line $i j, n$ represents the number of wires between the towers, $p_{i j, p, k}^{f a u l t}$ represents the failure rate of the tower $k$, and $p_{i j, c, k}^{f a u l t}$ represents the failure rate of the line $k . p_{i j, p, k}^{\text {fault }}$ and $p_{i j, c, k}^{f a u l t}$ will be presented in different forms under different extreme events. The model and expressions under extreme ice and snow weather and typhoons will be relatively discussed in Chapter 1.1-1.2.

\subsection{Model of line failure rate under extreme ice and snow weather}

The conductor should be able to bear additional ice load in addition to its own gravity load under extreme snow and ice weather. Under no wind and even load conditions, the ice load on the conductor can be expressed as [8]

$$
F_{c}^{i c e}=9.82 \times 10^{-9} \rho_{\text {ice }} \pi d_{\text {ice }}\left(D+d_{\text {ice }}\right) L_{p}
$$

Where $F_{c}^{i c e}$ represents the ice load on the wire, $\rho_{i c e}$ is the icing density, $D$ is the wire diameter, $L_{p}$ is the vertical span of the tower, and $d_{i c e}$ is the icing thickness. The icing thickness is related to the accumulation effect of ice and snow. In actual operation, the forecast usually first take meteorological factors into account, and consider the forecast error as normally distributed. $\rho_{i c e}$ and $d_{\text {ice }}$ are also related to the horizontal and vertical coordinates of the wire position when the load is uneven.
The total load borne by the wire can be expressed as

$$
F_{c}=G_{c}+F_{c}^{i c e}
$$

Where $G_{c}$ represents the gravity load of the wire.

The tower load in extreme snow and ice weather is generally composed of the horizontal tension applied by the transmission line, the longitudinal load of the icecovered line and the horizontal wind load. To simplify the study, only the longitudinal load of the ice-covered line on the tower under no wind conditions is considered. The longitudinal load on the tower can be expressed as [9]

$$
F_{p}=F_{c}^{i c e, p u}\left(\left(\frac{l_{1}}{2}+\frac{f_{1}}{F_{c}^{i c e, p u}} \frac{h_{1}}{l_{1}}\right)+\left(\frac{l_{2}}{2}+\frac{f_{2}}{F_{c}^{i c e, p u}} \frac{h_{2}}{l_{2}}\right)\right)
$$

Where $F_{p}$ represents the longitudinal load of the tower, $F_{c}^{i c e, p u}$ is the longitudinal load per unit length of the iced-covered conductor, $h_{1}$ and $h_{2}$ represent the suspension height difference between the two ends of the tower, $l_{1}$ and $l_{2}$ represent the span on both sides of the tower. $F_{c}^{i c e, p u}$ can be further expressed as [10]

$$
F_{c}^{i c e, p u}=0.0277 F_{c}^{i c e} \times\left(F_{c}^{i c e}+D\right)
$$

It can be considered that the failure rate of conductors and towers under the influence of extreme ice and snow weather has an exponential relationship with the load of the conductors and towers [11], that is:

$$
\begin{aligned}
& p_{i j, p}^{\text {fault }}=\alpha_{p} e^{\frac{\eta_{p}}{\beta_{p}}} \\
& p_{i j, c}^{\text {fault }}=k_{1} \alpha_{c} e^{\frac{\eta_{c}}{\beta_{c}}}+k_{2}\left|p_{i j}\right|
\end{aligned}
$$

Among them $\alpha_{p}, \alpha_{c}, \beta_{p}, \beta_{c}$ are all model coefficients, which can be obtained by fitting historical data. $\eta_{c}$ and $\eta_{p}$ relatively represent the longitudinal icing load rate of lines and towers, which can be expressed as

$$
\eta_{c}=\frac{F_{c}^{i c e}}{M_{c}}, \eta_{p}(t)=\frac{F_{p}}{M_{p}}
$$

$M_{c}$ and $M_{p}$ represent the design load, which are the rated parameter of the conductor and the tower. The failure rate of the conductor under the extreme ice and snow weather will also rise with the increase of the heavy load degree of the tidal current. $k_{1}$ and $k_{2}$ represent the weight coefficient corresponding to the line icing load and power flow in the line failure rate, which can be given by the engineer based on the operating experience. Moreover, $k_{1}$ and $k_{2}$ should meet the following condition:

$$
k_{1}+k_{2}=1
$$




\subsection{Model of line failure rate under typhoon weather}

The condition for wire breakage under typhoon is that the maximum carrying capacity of the wire under wind tension, namely the tensile strength, is less than the stress exerted by the typhoon on the cross section of the wire, the formula is expressed as

$$
\sigma_{l}<\sigma_{g}
$$

It is generally believed that the tensile strength of the wire is normally distributed. The probability distribution can be expressed as

$$
f\left(\sigma_{l}\right)=\frac{1}{\sqrt{2 \pi} \delta_{l}} e^{-\frac{1}{2}\left(\frac{\sigma_{l}-\mu_{l}}{\delta_{l}}\right)^{2}}
$$

Among them, $\mu_{l}$ and $\delta_{l}$ respectively represent the expectation and standard deviation of the normal distribution, which can be obtained based on the wire manufacturing manual and actual operating experience.

The stress imposed by the typhoon on the conductor cross-section $\sigma_{g}$ is proportional to the total load of the conductor [6].

$$
\sigma_{g}=k_{g} F_{c}
$$

Where $k_{g}$ is the load factor. The total load of the conductor under the influence of typhoon includes two parts, namely the lateral load $F_{c}^{\text {wind }}$ under the action of wind and the longitudinal load $G_{c}$ under the action of gravity. According to the vector nature of the load, it can be obtained

$$
\begin{aligned}
& F_{c}=\sqrt{\left(F_{c}^{\text {wind }}\right)^{2}+\left(G_{c}\right)^{2}} \\
& \text { And, } \\
& G_{c}=m g
\end{aligned}
$$

Wind load can be expressed as a function of wind force and wind direction, as shown in equation (15).

$$
F_{c}^{\text {wind }}=k_{w} \frac{V^{2}}{1.6} D \sin ^{2} \theta
$$

Where $V$ is the typhoon wind speed, $\theta$ is the angle between the wind direction and the line direction, $k_{w}$ is the coefficient which can be obtained by fitting.

The wind speed under the action of typhoon is more accurately fitted by the Batts model, which can be expressed as:

$$
V=\left\{\begin{array}{l}
V_{R_{\max }} \frac{r}{R_{\max }}, r \leq R_{\max } \\
V_{R_{\max }}\left(\frac{R_{\max }}{r}\right)^{0.7}, r>R_{\max }
\end{array}\right.
$$

Among them, $R_{\max }$ represents the maximum wind speed radius, $V_{R_{\max }}$ represents the wind speed at the corresponding location $R_{\max }$, and $r$ represents the distance from the wire to the center of the typhoon. $R_{\text {max }}$ can also be expressed as a function of the air pressure difference between the center of the typhoon and the periphery. The specific expression can be found in literature [10].

In summary, based on equations (10) and (11), the failure rate of conductors under the action of typhoon can be obtained in equation (17).

$$
p_{i j, c}^{\text {fault }}=\int_{0}^{\sigma_{g}} \frac{1}{\sqrt{2 \pi} \delta_{l}} e^{-\frac{1}{2}\left(\frac{\sigma_{l}-\mu_{l}}{\delta_{l}}\right)^{2}} d\left(\sigma_{l}\right)
$$

The condition for tower breakage under typhoon is that the maximum resistance of the tower to bending, namely the bending strength, is less than the bending moment at the root of the tower under the action of wind, which can be presented in formula form as

$$
M_{p}<M_{T}
$$

It can also be considered that the bending strength of the tower is normally distributed, and its probability can be expressed as (19):

$$
f\left(M_{p}\right)=\frac{1}{\sqrt{2 \pi} \delta_{p}} e^{-\frac{1}{2}\left(\frac{M_{p}-\mu_{p}}{\delta_{p}}\right)^{2}}
$$

Among them, $\mu_{p}$ and $\delta_{p}$ respectively represent the expectation and standard deviation of the normal distribution, which can be obtained based on the tower manufacturing manual and actual operating experience.

Finally, the failure rate of towers under typhoon is shown in equation (20).

$$
p_{i j, p}^{\text {fault }}=\int_{0}^{M_{T}} \frac{1}{\sqrt{2 \pi} \delta_{p}} e^{-\frac{1}{2}\left(\frac{M_{p}-\mu_{p}}{\delta_{p}}\right)^{2}} d\left(M_{T}\right)
$$

\section{Assessment method of HDG resilience}

\subsection{Model of line failure rate under typhoon weather}

Firstly, the generation process of the line fault set under extreme ice and snow weather and typhoon weather is simulated. Then, the set of failure rates of AC and DC distribution network lines is obtained based on the prediction of the characteristics and evolution of extreme natural disasters, as well as the line failure rate model under the influence of extreme natural disasters. There are many lines in the distribution network. It will cause huge calculation pressure and solving burden if the probability of corresponding line failure scenarios is calculated separately. Therefore, the Monte Carlo method is introduced to generate failure scenarios based on the sampling results. Taking typhoon weather as an example, the specific sampling steps can be expressed as

1) For any line $(i, j) \in \Omega_{L}$, the line failure rate under typhoon weather is $p_{i j}^{\text {fault }}$ according to Chapter 1.2. Generate a uniformly distributed random number $r$ in 
the interval $[0,1]$, then the line operating state $x_{i j}$ can be expressed as

$$
x_{i j}=\left\{\begin{array}{l}
0, r>p_{i j}^{\text {fault }} \\
1, r \leq p_{i j}^{\text {fault }}
\end{array}, \forall(i, j) \in \Omega_{L}\right.
$$

Traverse all lines of the AC/DC distribution network until the operating status of all lines is obtained, and the set of line operating status is obtained $\mathbf{X}_{1}$ 。

2) Repeat step 1 to get the set of $M$ line operating status $\mathbf{X}=\left[\mathbf{X}_{1}, \cdots, \mathbf{X}_{\mathbf{M}}\right]$. At this time, the actual operating state of the equivalent circuit of the $M$ scenario can be obtained by sampling.

\subsection{Resilience evaluation indexes of AC-DC hybrid distribution network}

The resilience of the distribution network reflects its ability to resist and deal with extreme events with small probability and high risk. In this paper, then, a comprehensive evaluation index system for resilience of AC-DC hybrid distribution network is proposed.

\subsubsection{Indexes of strain capability}

(1) Percentage of Schedulable Resources (PSR)

As an emergency resource, distributed energy resources in the AC-DC distribution network need to meet two conditions when it participates in post-disaster deployment and isolated island operation. The first one is that there are optimizable units, which means that the unit with high volatility can not be used as the main control power supply to support the islanding operation such as the renewable energy unit because it is difficult to accurately and effectively control the voltage and frequency independently. Second, it must have excellent regulation performance. Emergency resources must respond within a short time after the disaster to ensure the supply of important loads, so it has short start-up and shutdown time and rapid response. The proportion of dispatchable emergency resources measures the proportion of emergency resources in the distributed energy resources of AC-DC distribution network, and its formula can be expressed as Equation (25).

PSR $=\frac{\text { Emergency resources }}{\text { Total capacity of distibuted energy resources in }}$

$\overline{\text { Total capacity of distributed energy resources in distribution network }}$

(2) Emergency resource availability rate

The emergency resource supply rate measures the theoretical upper limit of the critical load ratio that can be supplied under ideal conditions only depending on emergency resources. It can be expressed as:

Emergency resource availability rate $=\frac{\text { Maximum power output of emergency resources }}{\text { Active power of the important load }}$

(3) Remote Control Feeder Switch Placement Rate (RCFSPR)

Remote control feeder switch is the key to adjust the topology of distribution network actively. Remote feeder switch placement rate is to measure the proportion of AC/DC lines with feeder switches.
RCFSPR of DC/AC lines $=\frac{\text { Number of DC/AC lines with feeder switch }}{\text { Total number of DC/AC distribution network lines }}$

(4) Connection Lines Supply Rate (CLSR)

Due to the limitation of the transmission power capacity and VSC capacity of the connection line, there is a certain upper limit of power support from normal distribution network to fault distribution network. The AC-DC connection line supply ratio is to measure the maximum theoretical support capacity from normal distribution network to fault distribution network, which can be expressed as:

CLSR of $\mathrm{AC} / \mathrm{DC}=\frac{\text { Maximum trasmission active power of connection lines }}{}$ Active power of the important load

\subsubsection{Indexes of defence capability}

The number of fault lines is chosen to represent the defensive force of distribution network, which can be specifically expressed as:

$$
\text { The expectation of DC/AC fault lines' number }=E\left(\sum_{(i, j) \in \Omega_{L}^{D C / A C}} x_{i j}\right)
$$

\subsubsection{Indexes of recovery capability}

(1) Power loss time of the important load

The power loss time of important loads is to measure the time when the power supply of important loads in the distribution network is short during the occurrence of extreme events. That is, the elapsed time between the occurrence of the fault and the repair of the line.

(2) Power loss rate of the important load

The important load loss rate is to measure the proportion of critical load reduction to critical load during the occurrence of extreme events.

Power loss rate of the imporatant load $=\sum_{i, t} \Delta p_{i, t}^{D} \Delta t / \sum_{i, t} p_{i, t}^{D} \Delta t, \forall i \in \Omega_{B}^{I p}, \forall t$

Among these, $\Delta p_{i, t}^{D}$ is to represent the amount of load shedding, and $\Omega_{B}^{I p}$ is the set of nodes with the important load.

(3) Total loss of distribution network load shedding

The load shedding loss of the distribution network reflects the economic loss caused by extreme events to the distribution network, which is equal to the product of unit load shedding cost and load shedding.

Total loss of distribution network load shedding $=\sum_{i, t} c_{i, l}^{L s} \Delta p_{i, t} \Delta t, \forall i \in \Omega_{B}, \forall t$

\subsection{Resilience assessment process of AC/DC hybrid distribution network}

The approximate ideal solution ranking method (TOPSIS) is used to comprehensively evaluate the resilience indexes of AC/DC hybrid distribution network. Among them, the objective weight of each index can be obtained based on the entropy weight method. The principle of entropy weight method can be found in literature [10] for details, and the principle and solving steps of TOPSIS algorithm 
can be found in literature [11]. Fig. 1 shows the toughness assessment process of AC-DC hybrid distribution network.

\begin{tabular}{|c|c|c|c|c|c|}
\hline \multirow{4}{*}{ Start } & \multirow{4}{*}{$\begin{array}{l}\text { If the type of extreme } \\
\text { events is natural } \\
\text { disasters (such as } \\
\text { snow disasters and } \\
\text { typhoon) }\end{array}$} & & & & \\
\hline & & \multirow{3}{*}{$\begin{array}{l}\text { The characteristics } \\
\text { and evolution } \\
\text { rules of natural } \\
\text { disasters are } \\
\text { predicted. }\end{array}$} & \multirow{3}{*}{\multicolumn{2}{|c|}{$\begin{array}{l}\text { The failure rate of } \\
\text { distribution network } \\
\text { line is obtained based } \\
\text { on the failure rate } \\
\text { model. }\end{array}$}} & \multirow{4}{*}{$\begin{array}{c}\text { Several sets of } \\
\text { failure scenarios are } \\
\text { generated based on } \\
\text { Monte Carlo } \\
\text { sampling method. }\end{array}$} \\
\hline & & & & & \\
\hline & & & & & \\
\hline \multirow[b]{2}{*}{ End } & $\begin{array}{l}\text { The resilience of AC- } \\
\text { DC hybrid distribution }\end{array}$ & $\begin{array}{l}\text { Based on entropy } \\
\text { weight method, }\end{array}$ & & $\begin{array}{l}\text { The expected } \\
\text { value of each }\end{array}$ & \\
\hline & $\begin{array}{l}\text { network is evaluated } \\
\text { comprehensively. }\end{array}$ & $\begin{array}{l}\text { objective weight is giv } \\
\text { to each resilience ind }\end{array}$ & & $\begin{array}{l}\text { resilience index } \\
\text { is obtained. }\end{array}$ & \\
\hline
\end{tabular}

Figure 1. The flowchart of resilience assessment of HDG.

\section{Case study}

This paper takes typhoon as an example to analyse the resilience assessment method of AC-DC hybrid distribution network under the influence of typhoon. The AC-DC hybrid distribution network composed of IEEE 33-node AC distribution network system and 9-node DC distribution network system is selected as the example system in this paper, in which the AC distribution network and DC distribution network topology can be seen in Figure 2 and Figure 3. The text of your paper should be formatted as follows:

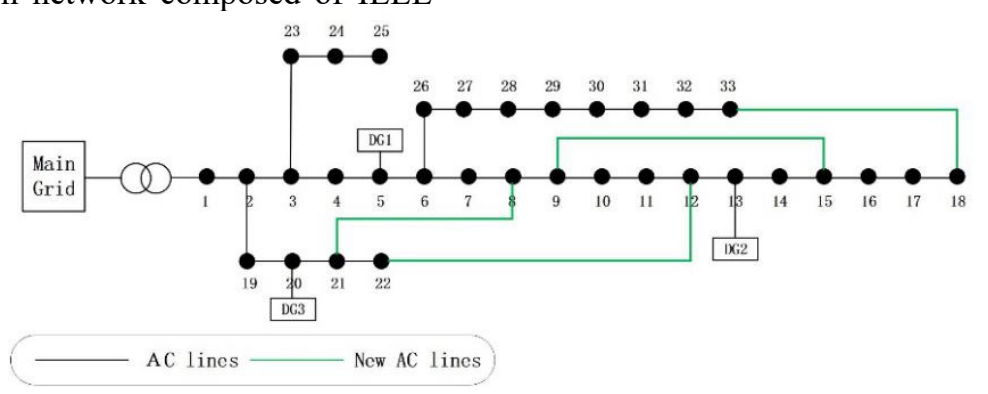

Figure 2. Topology of improved IEEE-33 node meshed AC distribution grid.

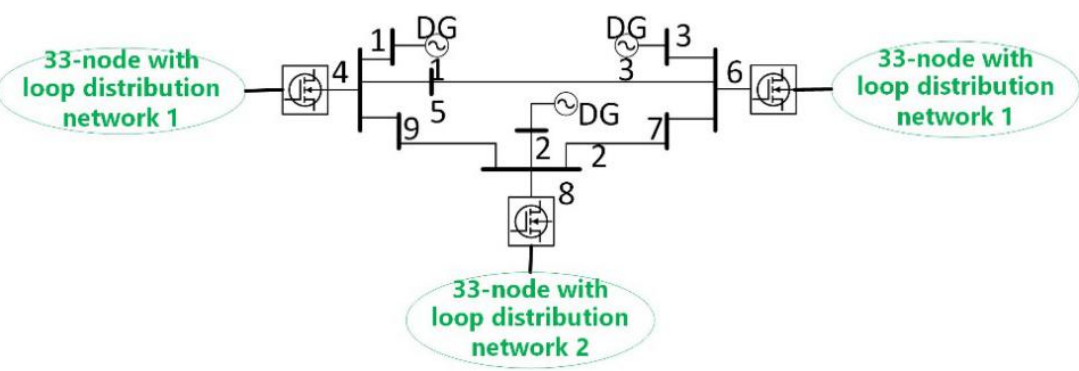

Figure 3. System structure of HDG in the case

The influence of the distance between the distribution network line and the typhoon centre is not considered temporarily. Based on the line failure rate model under the influence of typhoon constructed in this paper and the relevant data in Reference [11], the failure rates of conductors and poles and towers corresponding to different wind levels can be obtained, as shown in Table 1. The set lines are all single loops, and the corresponding line failure rates can be obtained. Based on Monte Carlo sampling, 50 fault scenarios under different wind levels were generated. Finally, the calculation results of each indicator proposed in Section 2.2 and the comprehensive evaluation results of toughness of AC-DC hybrid distribution network under different wind levels can be seen in Table 2. The second column in the table represents the weight of each indicator calculated based on entropy weight method.

Table 1. Result of resilience assessment indicators of HDG under different wind scales.

\begin{tabular}{ccccc}
\hline Wind Scale & Median wind speed & $\begin{array}{c}\text { Conductor failure } \\
\text { rate }\end{array}$ & Tower failure rate & Line failure rate \\
\hline 0 & 0 & 0 & 0 & 0 \\
6 & 12.3 & 0.031 & 0 & 0.031 \\
11 & 30.5 & 0.447 & 0.015 & 0.463 \\
12 & 34.8 & 0.812 & 0.129 & 0.857
\end{tabular}




\begin{tabular}{ccccc}
13 & 39.2 & 0.930 & 0.575 & 0.987 \\
14 & 43.8 & 1 & 1 & 1 \\
\hline
\end{tabular}

Table 2. Result of resilience assessment indicators of HDG under different wind scales.

\begin{tabular}{cccccccc}
\hline Resilience indexes & Index & Wind & Wind & Wind & Wind & Wind & Wind \\
weight & Scale 0 & Scale 6 & Scale 11 & Scale 12 & Scale 13 & Scale 14 \\
\hline Percentage of schedulable resources & 0.117 & 0.608 & 0.608 & 0.608 & 0.608 & 0.608 & 0.608 \\
Emergency resource availability rate & 0.151 & 0.251 & 0.251 & 0.251 & 0.251 & 0.251 & 0.251 \\
Remote control feeder switch placement & 0.133 & 0 & 0 & 0 & 0 & 0 & 0 \\
rate & 0.021 & 0.507 & 0.507 & 0.507 & 0.507 & 0.507 & 0.507 \\
AC/DC connection lines supply rate & 0.152 & 0 & 2.26 & 33.18 & 62.54 & 71.08 & 72 \\
$\quad$ The number of fault lines & 0.089 & 6 & 6 & 6 & 6 & 6 & 6 \\
$\begin{array}{c}\text { Power loss time of the important load(h) } \\
\text { Power loss rate of the important load }\end{array}$ & 0.155 & 0 & 0.018 & 0.395 & 0.822 & 0.997 & 1 \\
$\begin{array}{c}\text { Total loss of distribution network load } \\
\text { shedding (10,000Yuan) }\end{array}$ & 0.182 & 0 & 1.36 & 221.38 & 425.62 & 501.58 & 505.23 \\
$\begin{array}{c}\text { Comprehensive assessment results of } \\
\text { resilience }\end{array}$ & $/$ & 1 & 0.981 & 0.568 & 0.157 & 0.008 & 0 \\
\hline
\end{tabular}

As can be seen from the results in the table, typhoon weather mainly affects HDG's defence and resilience indexes. Because there is no resource allocation strategy involved, the toughness index in the pre-disaster defence stage remains unchanged in all scenarios. With the increase of wind grade, the comprehensive evaluation result of HDG toughness is getting lower and lower. When the wind force reaches level 11, the power loss rate of the important load reaches 0.395 , and the comprehensive toughness evaluation result decreases $43.2 \%$ compared with the maximum value. However, after the wind level officially reached typhoon level 12, the power loss rate of important loads reached 0.8 , and the comprehensive toughness evaluation result was only 0.157 . When the wind level reaches level 14, the distribution network lines and equipment will be all out of service. Therefore, the impact of typhoon weather on HDG is huge and cannot be ignored, and relevant strategies for improving resilience before, during and after disasters are needed to improve the power distribution network's ability to withstand typhoon weather.

\section{Conclusion}

In this paper, a complete and previous resilience assessment process for AC-DC hybrid distribution network considering extreme event characteristics is proposed. First of all, for natural disasters in extreme events, the different generation modes of fault sets of ACDC distribution network lines under the influence of different natural disasters are fully considered, and a line failure rate model under the corresponding influence is built. Several groups of fault scenarios are generated based on Monte Carlo sampling method. Then, based on the three core characteristics of resilience, namely, capability of strain, defence and recovery, the resilience assessment index system of AC/DC hybrid distribution network was constructed, and the objective weight of each index was obtained based on entropy weight method. Finally, the comprehensive evaluation results of resilience in $\mathrm{AC} / \mathrm{DC}$ hybrid distribution network were obtained based on TOPSIS algorithm. The research results of this chapter will serve as the theoretical basis for analysing the current resilience state of distribution network and evaluating the effect of resilience improvement strategy.

\section{Acknowledgments}

This research was financially supported by Science and Technology Project of SMEPC (B30921200000), and the project name is Research on Active Distribution Network Planning and Operation Method for Resilience Improvement.

\section{References}

1. Liu Xiaolin, Wang Xu, Jiang Chuanwen, Gong Kai, Xiong Zhan. Distributed Optimal Scheduling Method for AC/DC Hybrid Distribution Network Considering VSC Operational Constraints [J].Power System Technology, 211,45(03):1089-1101.

2. X. Wang, Z. Li, M. Shahidehpour* and C. Jiang, "Robust line hardening strategies for improving the resilience of distribution systems with variable renewable resources," IEEE Transactions on Sustainable Energy, vol. 10, no. 1, pp. 386-395, Jan. 2019.

3. Ruan Qiantu, XIE Wei, XU Yin, et al.Concepts and Key Characteristics of Resilient Power Grid. Proceedings of the CSEE, 2020, 40(21): 6773-6784.

4. Wang Shouxiang, Huang Renshan, Pan Zhixin, Wang Jianming. Construction and evaluation method of elastic resilience index of distribution network under extreme snow and ice weather $[\mathrm{J}]$. High Voltage Technology, 2020, 46(1): 123-132.

5. Y. Wang, C. Chen, J. Wang and R. Baldick. Research on Resilience of Power Systems Under Natural Disasters-A Review[J]. IEEE Transactions on Power Systems, 2016, 31(2): 1604-1613. 
6. Zhou Xiaomin, Ge Shaoyun, Li Teng, Liu Hong. Research on Toughness Analysis Method and Hoisting Measures of Distribution Network under Extreme Weather Conditions. Proceedings of the CSEE, 2018, 38(2): 505-513+681.

7. S. Ma, B. Chen and Z. Wang. Resilience Enhancement Strategy for Distribution Systems Under Extreme Weather Events[J]. IEEE Transactions on Smart Grid, 2018, 9(2): 1442-1451.

8. Wang Shouxiang, Huang Renshan, Pan Zhixin, Wang Jianming. Construction and evaluation method of elastic resilience index of distribution network under extreme snow and ice weather [J]. High Voltage Technology, 2020, 46(01): 123-132.

9. Xia Yongwei. Research on Toughness Evaluation and Upgrading Method of Distribution Network Considering Critical Loads [D]. Hunan University, 2019.

10. SONG Junying, HE Cong, LI Xinran, et al.Journal of Power Systems Automation, 2019, 43(20): 65-76. (in Chinese)

11. M. Alhabo and L. Zhang. Multi-Criteria Handover Using Modified Weighted TOPSIS Methods for Heterogeneous Networks[J]. IEEE Access, 2018, 6, vol. $6,40547-40558$. 